\title{
BMJ Open Bike skills training for children with cerebral palsy: protocol for a randomised controlled trial
}

\author{
Rachel Toovey, ${ }^{1,2,3}$ Adrienne R Harvey, ${ }^{2,4,5}$ Jennifer L McGinley, ${ }^{1}$ \\ Katherine J Lee, ${ }^{5,6,7}$ Sophy T F Shih, ${ }^{8}$ Alicia J Spittle ${ }^{1,9}$
}

To cite: Toovey R, Harvey AR, McGinley JL, et al. Bike skills training for children with cerebral palsy: protocol for a randomised controlled trial. BMJ Open 2018;8:e019898. doi:10.1136/ bmjopen-2017-019898

- Prepublication history and additional material for this paper are available online. To view these files, please visit the journal online (http://dx.doi. org/10.1136/bmjopen-2017019898).

Received 3 October 2017 Revised 28 November 2017 Accepted 6 December 2017
Check for updates

For numbered affiliations see end of article.

Correspondence to Associate Professor Alicia J Spittle;

aspittle@unimelb.edu.au

\section{ABSTRACT}

Introduction Two-wheel bike riding can be a goal for children with cerebral palsy (CP) and a means of participating in physical activity. It is possible for some children with CP to ride a two-wheel bike; however, currently far fewer can ride compared with their typically developing peers. Evidence supports training targeted towards goals of the child with CP and their family; yet there is little evidence to guide best-practice bike skills training. Task-specific training may lead to attainment of two-wheel bike-specific goals. This study aims to determine if a novel task-specific approach to training two-wheel bike skills is more effective than a parent-led home programme for attaining individualised two-wheel bike-specific goals in independently ambulant children with CP aged $6-15$ years.

Methods and analysis Sixty eligible children with $\mathrm{CP}$ (Gross Motor Function Classification System levels I-II) aged 6-15 years with goals relating to riding a two-wheel bike will be randomised to either a novel task-specific centre-based group programme (intervention) or a parentled home-based programme (comparison), both involving a 1-week intervention period. The primary outcome is goal attainment in the week following the intervention period (T1). Secondary outcomes include: goal attainment and participation in physical activity at 3 months postintervention (T2) and bike skills, attendance and involvement in bike riding, self-perception and functional skills at T1 and T2. Economic appraisal will involve costeffectiveness and cost-utility analyses. Adherence of clinicians and parents to the intervention and comparison protocols will be assessed. Linear and logistic regression will be used to assess the effect of the intervention, adjusted for site as used in the randomisation process.

Ethics and dissemination This study was approved by the Human Research and Ethics Committees at The Royal Children's Hospital (\#36209). Results will be disseminated via peer-reviewed publications and conference presentations.

Trial registration number NCT03003026; Pre-results.

\section{INTRODUCTION}

Cerebral palsy (CP) is the most common cause of childhood physical disability affecting 1 in 500 births. $^{1}$ It is a group of disorders of the development of movement and posture, causing activity limitations that are attributed
Strengths and limitations of this study

- To our knowledge, this will be the first adequately powered randomised controlled trial to evaluate the effectiveness of a novel task-specific bike skills training programme for attaining bike-specific goals in children with cerebral palsy.

- The range of secondary outcomes will allow for assessment of the effects of training bike skills on a range of activity and participation outcomes.

- Assessment of fidelity will enable evaluation of the extent to which clinicians and families adhere to the intervention and comparison group protocols.

- The economic appraisal will be useful for future policy and decision-making.

- Due to the nature of the intervention, clinicians delivering the interventions and participants will not be blind to allocation.

to non-progressive disturbances occurring in the developing fetal or infant brain. ${ }^{2}$ Children with CP participate less in physical and recreational activities than their typically developing peers, ${ }^{3}$ putting them at increased risk of poor health and disease in adulthood. ${ }^{4}$ Effective means of engaging children with $\mathrm{CP}$ are required to improve physical activity patterns in this population, and evidence supports training targeted towards goals of the child and their family. ${ }^{5}$ Bike riding is a common activity for families ${ }^{6}$ and may be an effective means of involving ambulant children with $\mathrm{CP}$ in physical activity that is enjoyable and meaningful to them.

The Gross Motor Function Classification System $(\mathrm{GMFCS})^{7}$ uses five levels (I-V) to classify children with $\mathrm{CP}$ according to their level of motor function. Children classified as levels I-II are independently ambulant with or without hand-held devices. Far fewer ambulant children with CP (GMFCS I-II) can ride a two-wheel bike at any given age compared with their typically developing peers, and if they do, they learn later in life. However, it is possible for children with CP at GMFCS levels 
I and II to learn to ride and the majority who do so, learn at home with their parents. ${ }^{8}$

Physiotherapists and occupational therapists routinely implement training to improve motor skills in children with CP. However, there is very little specific evidence to guide best practice in training of bike riding skills. The studies that do exist specific to children with $\mathrm{CP}$ have been conducted on stationary bikes ${ }^{9-11}$ with no evidence to suggest this translates to riding a two-wheel bike in the community. Furthermore, the current practices of Australian physiotherapists and occupational therapists for training two-wheel bike skills in children with CP are not well understood. Importantly, there does not appear to be a standard or usual care.

The development and testing of approaches to training bike skills is required to provide clinicians and families with evidence-based guidance when working with children with CP with two-wheel bike-specific goals. Strong evidence exists for task-specific training to improve general upper limb function in this population ${ }^{512}$ and gross motor skills in adults following stroke. ${ }^{13}$ Task-specific training involves practice of context-specific tasks where the intervention focuses on the skills needed for a task (s).$^{14}$ It is informed by principles of motor learning ${ }^{15}$ and dynamic systems theory ${ }^{16}$ and involves a dynamic interaction between the task, the child and the environment to achieve a motor skill in a task-specific context. ${ }^{17}$ Evidence for task-specific training to improve gross motor skills in ambulant children with CP exists, ${ }^{18} 19$ but is currently limited by poor study methodology and intervention heterogeneity. ${ }^{20}$ An intensive task-specific approach to training bike skills has seen promising outcomes in a group setting at the two main paediatric rehabilitation settings in Victoria, Australia demonstrated through results from a small pilot case series $(n=5) .{ }^{21}$ While this clinical evidence supports the safety and feasibility of task-specific training in bike riding in a group setting, an adequately powered study with a comparison group is required to ascertain the effectiveness of such an approach.

\section{Objectives}

The primary objective of this study is to determine if a novel task-specific approach to training bike skills is more effective than a parent-led home programme in ambulant children with CP (GMFCS I-II) aged 6-15 years, for attaining individualised two-wheel bike-specific goals immediately following the intervention period (T1).

The secondary objectives of this study are:

1. To determine if a novel task-specific approach to training bike skills is more effective compared with a parent-led home programme in children with $\mathrm{CP}$ (GMFCS I-II) aged $6-15$ years on:

a. Goal attainment at 3 months following the intervention (T2);

b. Acquiring and retaining two-wheel bike skills at T1 and $\mathrm{T} 2$;

c. Functional skills at T1 and T2;

d. Physical activity behaviour at T2; e. Self-perception at T1 and T2;

f. Self-perceived bike riding competence at $\mathrm{T} 1$ and T2.

2. To compare attendance and involvement in bike skills training between the intervention and comparison groups during the intervention and follow-up periods.

3. To conduct an economic appraisal, involving assessment of quality of life, of the intervention compared with the comparison programme.

4. To examine clinician and parent fidelity with delivery of both group protocols.

\section{METHODS AND ANALYSIS \\ Design}

Assessor-blinded, parallel-group, randomised controlled, multicentre, superiority trial comparing a novel task-specific approach to a parent-led home programme for training bike skills. This study involves a 1-week intervention period and 3-month follow-up period (figure 1).

\section{Setting}

The study will be conducted through the Victorian Paediatric Rehabilitation Service (VPRS: a state-wide rehabilitation service for children with rehabilitation goals including children with CP) at The Royal Children's Hospital and Monash Children's Hospital in Melbourne, Australia.

\section{Participants}

Sixty participants will be recruited from the Victorian Cerebral Palsy Register (VCPR: a register of children with $\mathrm{CP}$ who were born in Victoria or receive health services in Victoria) and the VPRS. Approximately 30 children will be randomised to the intervention group and 30 children will be randomised to the comparison group (figure 1). Each participant must meet all of the inclusion criteria and none of the exclusion criteria to be enrolled in this study (box).

\section{Recruitment procedures}

Participants will be identified and recruited through the VCPR and the VPRS at The Royal Children's Hospital and Monash Children's Hospital. The study will also be advertised on the National Health and Medical Research Council Centre of Research Excellence in Cerebral Palsy (CRE-CP) newsletter and website.

\section{Victorian Cerebral Palsy Register}

Within this register, it is recorded whether parents or primary care givers have consented to being contacted for research purposes. Invitations to participate in the study will be sent by VCPR staff to potentially eligible participants whose parents/primary care givers have provided consent by email or letter including a full participant information and consent form. Families will have the opportunity to contact the VCPR to request that their contact details not be passed onto the study team for follow-up and screening for eligibility which will occur by email and phone. 


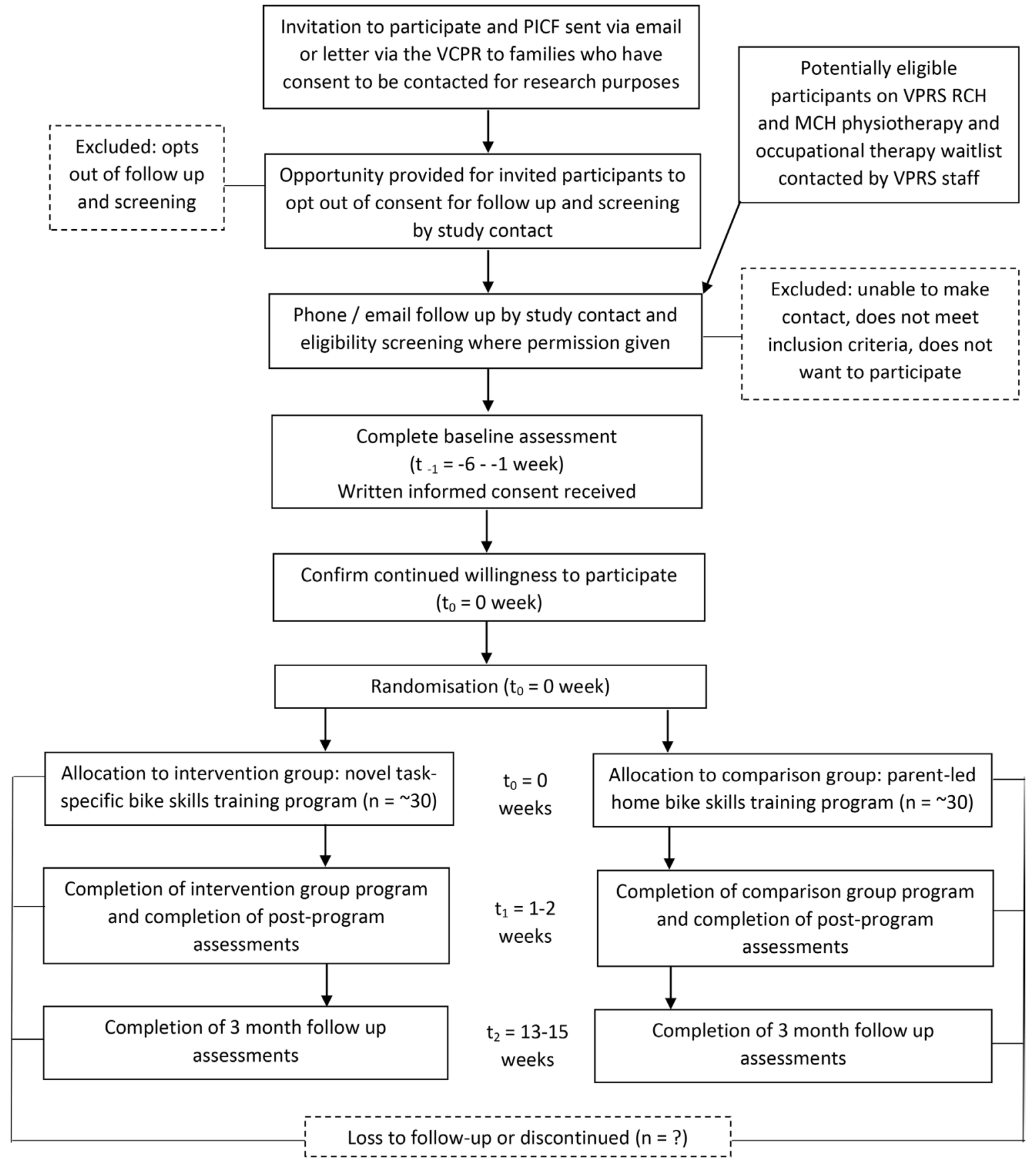

Figure 1 Study timeline. $t_{-1}$, enrolment and baseline assessment time point; $t_{0}$, randomisation and allocation time point; $t_{1}$, first follow-up assessment time point; $t_{2}$, final follow-up assessment time point; MCH, Monash Children's Hospital; PICF, participant information and consent form; RCH, The Royal Children's Hospital; VCPR, Victorian Cerebral Palsy Register; VPRS, Victorian Paediatric Rehabilitation Service.

\section{The Victorian Paediatric Rehabilitation Service}

Waitlists for services and clinics at VPRS sites at The Royal Children's Hospital and Monash Children's Hospital, will also be used to identify potentially eligible participants. A VPRS clinician will contact the parents of potentially eligible participants as per respective VPRS site physiotherapy waitlists. Potentially eligible participants who attend VPRS clinics at both hospitals during the recruitment period but are not yet on the respective VPRS physiotherapy waitlists will also be identified by VPRS clinicians. Interested families will be given the study contact's details or permission will be sought by the VPRS clinicians to pass their contact information on to the study contact for screening and follow-up.

The Centre for Research Excellence in Cerebral Palsy website and e-newsletter

An advertisement inviting eligible families to participate in the study will be posted on a parent, clinician and researcher website for the management and treatment of CP (http://www.cre-cp.org.au) and in the website's e-newsletter during the recruitment period. 


\section{Box Inclusion and exclusion criteria}

\section{Inclusion criteria}

- Is between age 6 and 15 years at the time of randomisation.

- Has cerebral palsy as determined by the Victorian Cerebral Palsy Register (VCPR) or in writing from the child's general practitioner or paediatrician if not on the VCPR.

- Is independently ambulant without aids (Gross Motor Function Classification System I-II).

- Has goals related to improving two-wheel bike skills.

- Has a primary caregiver available to participate in the intervention.

- Has a legally acceptable representative capable of understanding the informed consent document and providing consent on the participant's behalf.

- Lives in Victoria or close to the Victorian border in New South Wales and receives health services in Victoria.

- Has access to an appropriately sized two-wheel bike and helmet.

- Has medical clearance to participate in the study from the child's general practitioner, paediatrician or paediatric specialist.

- Participant and primary caregiver able to understand English.

\section{Exclusion criteria}

- Has a moderate-to-severe intellectual impairment.

- Has a dual diagnosis with another developmental disability or medical condition that may impact on their ability or safety to train two-wheel bike skills.

- Had musculoskeletal surgery, or other major surgery including insertion of a baclofen pump that may affect their physical ability in the 6 months prior to randomisation.

- Had Botulinum toxin-A injections to the lower limbs and/or upper limbs in the 6 months prior to randomisation.

\section{Baseline study visit}

Eligible participants will be enrolled in the study at the baseline (T0) assessment visit up to 6 weeks prior to the intervention period. Written informed consent will be obtained prior to performing any assessments and randomisation by the Principal Investigator or trained outcomes assessor. The following will be collected at the T0 assessment (see also supplementary appendix 1):

- Age, intellectual impairment (if any) and description of the CP including: topography, motor type, GMFCS level and Manual Ability Classification System level.

- Previous time spent practising bike skills on average per week or month since commencement of bike skills practice.

- Parent rated importance of their child attaining their goals, competence of their own bike skills and family interest in bike riding on a five-point scale.

- Family social risk as measured by a questionnaire comprised six questions regarding social status including family structure, education of primary caregiver, occupation of primary income earner, employment status of primary income earner, language spoken at home and maternal age at birth. ${ }^{22}$

- Goals will be set by the child, parent and outcomes assessor together using the Goal Attainment Scale (GAS) ${ }^{23}$
Baseline data for secondary outcomes will be collected including: two-wheel bike skills, functional skills, physical activity behaviour, self-perception, self-perceived two-wheel bike riding competence and health-related quality of life as assessed by the measures detailed below under the 'Primary and secondary outcome measures' section.

\section{Randomisation and blinding}

A statistician not directly involved in the study will prepare the randomisation schedule using computer-generated block randomisation with variable block sizes. Randomisation will be stratified by site. The statistician will generate opaque, numbered, sealed envelopes according to the randomisation schedule. In the week prior to the intervention period the participant will be allocated a sequential study number within the appropriate strata. Participants will then be randomised by a study investigator not involved in assessment procedures who will open the envelopes and inform participants of their allocation via phone or email. Participants who are already known to either site will be randomised within that site, otherwise families will be randomised within a site based on family preference or home location. The outcome assessors will be blind to group allocation, but it will not be possible to blind the treating clinicians or participants.

\section{Details of the intervention and comparison}

The intervention: novel task-specific bike skills training programme Participants randomised to the intervention group will participate in a novel bike skills training programme conducted over three consecutive days, with a further 4 days for practising the learnt skills at home (7-day intervention period). This approach involves seven key components:

1. Task-specific: training will be informed by the dynamic systems theory and principles of motor learning. The dynamic interaction between systems including the task, the child and the environment is considered to achieve motor skills in a task-specific context. ${ }^{16}$ Each of these systems is considered at the stages of the motor learning process. Initially, new motor tasks are scaffolded, so that the participant will always actively complete at least part of the task. This may involve task demonstration or physical guidance. As performance improves, the task and/or environment is altered to encourage problem solving and increase the motor challenge. This may include modifying the bike (eg, seat height, location of the brakes, basic straps for hand or feet) and reducing the physical guidance in order to achieve each progression of the skill/s. Once a motor skill is acquired, variability and randomness of practice in terms of task difficulty and environmental challenge will be introduced to increase the complexity and generalisability of the skill. ${ }^{24}$ Overall practice will be repetitive, progressive, variable and favour whole skill practice rather than part practice. ${ }^{24}$ The amount and type of feedback from the trainer 
will be guided by participant preference, and will focus on knowledge of results or performance for each new skill, ${ }^{25}$ for example, getting on and off the bike. Participants will use their own two-wheel bike without training wheels and helmet where possible. Cones or markers will be used as visual cues for skill practice.

2. Group-based: training will be delivered to groups of up to six participants. There is evidence to suggest group-based rehabilitation programmes improve functional skills, self-perceived performance and cost-effectiveness of treatment as much, or more than individual therapy. ${ }^{26}$

3. Clinician-mediated: each programme will be conducted by at least one physiotherapist and one other clinician (physiotherapist, occupational therapist or allied health assistant). There will be a minimum ratio of one clinician to three child participants in each group. All clinicians will be employed by VPRS and will undertake $6-8$ hours training in the intervention protocol in the 4 months prior to delivering the intervention. The same two clinicians will lead the 3 days of each programme where possible.

4. Intensive: each programme will run for 2 hours/ day over three consecutive days during 1 week of the school holiday period. This intensity is supported by motor learning literature, in particular the benefits of repetitive practice in the skill acquisition phase. ${ }^{27}$ This intensity allows for repetitive practice, ${ }^{18}$ including repetitive practice in the home environment following the programme and has been supported by parent evaluation of the intensive programme delivered as part of the pilot case series. ${ }^{21}$ Breaks from physical activity will be offered at least every $30 \mathrm{~min}$ and families can request additional rests. Participants will also be given a home programme of one to three bike skills practice exercises following each session and encouraged to practice these up to $30 \mathrm{~min} /$ day during the week-long intervention period and three to five bike skills to practice when able in the 3-month follow-up period.

5. Goal-directed: evidence suggests interventions that are goal-directed improve gross motor function more than those that are not. ${ }^{28}$ Goal setting is a key component of paediatric rehabilitation and has been well established in the literature. ${ }^{29}$ GAS will be used as an outcome measure and as a process for setting goals related to bike skills training. Clinicians delivering the intervention will be aware of each participant's goals, which will be used to provide individualised opportunities for problem solving and drive the movements required to meet the task demands. ${ }^{30}$

6. Parent or caregiver involvement: at least one parent or caregiver will be required to attend each session of the programme. Parent involvement and education is recognised as a key component in family centred practice. ${ }^{31}$ It facilitates a partnership between the clinician and parent towards achieving the child's goal. Parents will be coached by the clinician during the 3-day intervention regarding approaches to motor learning, including gradually increasing the difficulty of the task while ensuring this intersects with success. Parents will be provided verbal guidance regarding strategies and safety of practice in the home environment. ${ }^{24}$

7. Ecological setting: when possible the programme will be conducted in outdoor recreation or community reserves at or in close proximity to the rehabilitation service. This aligns with dynamic systems theory and task-specific training in terms of the role the environment has in promoting motor learning. Different surfaces and gradients will be available to individualise the environment based on each participant's stage of motor learning and to promote successful problem solving. All programme settings will be conducted away from roads and busy public spaces. Participants will be encouraged to practice outside of the programme in similar environments and advised to avoid practice on roads, busy bike paths or other risky environments during the intervention and follow-up periods.

\section{The comparison: parent-led home bike skills training programme} Current bike skills training for children with $\mathrm{CP}$ is not well understood. Given the lack of specific evidence, current practice is not likely to be uniform in approach, dosage or setting. While the majority of ambulant children with CP (GMFCS I-II) are currently not able to ride a two-wheel bike, many of those who can ride learnt in informal settings with their families. ${ }^{8}$ There also is evidence to support home-based therapy programme involving parent education for goal attainment in children with CP. ${ }^{5} 32$ Given this, it seemed appropriate that the comparison group for the intervention was a parent-led, home-based programme.

Participants randomised to the comparison group will receive written general information on training bike skills either in person or via email dependent on consenting and baseline assessment location. Families will receive this information at the start of the 1-week period of training during the school holidays. Parents will be encouraged to work with their child on two-wheel bike skills goals guided by the written information (available on request). This information involves:

1. Intensity: families will be encouraged to practice at least $30-45 \mathrm{~min}$ on each of the 7 days of the 1 -week period.

2. Safety: families will be encouraged to practice in settings away from roads and busy public spaces. They will also be advised to perform a risk assessment of the location prior to commencing. Information on appropriate weather and adequate hydration will also be included.

3. Appropriate bike and helmet fit: information regarding fitting the bike and helmet to the child for skill development, safety and potentially useful modifications will be provided. 
A trained VPRS physiotherapist will also telephone families in the comparison group between 3 and 5 days into the training period. The purpose of this phone call will be to inquire about how the family is managing with the training programme and to offer general advice regarding practice for the remaining 2-4days of the training period.

\section{Primary and secondary outcome measures}

Outcomes will be measured in the week following (T1) and 3 months (12-14 weeks) following (T2) the intervention period (see online supplementary appendix 1 ). Outcomes will be assessed by the Principal Investigator (RT) or a physiotherapist trained in the outcomes assessment, both blinded to group allocation.

The primary outcome, goal attainment at $\mathrm{T} 1$, will be measured using GAS, a criterion referenced tool for individualised and collaborative goal setting between the child, family and clinician. ${ }^{23} 2933$ GAS is commonly used in rehabilitation for children with CP because it is valid, ${ }^{33}$ reliable and responsive ${ }^{34}$ in this heterogeneous population. GAS will be facilitated by the blinded outcomes assessors, trained in administering GAS. Two to three individualised and measurable two-wheel bike-specific activity or participation goals per participant will be set at the baseline visit (T0). Six potential outcomes will be specified for each goal: -3 (deterioration), -2 (equal to start), -1 (less than expected), 0 (expected), 1 (somewhat more than expected), 2 (much more than expected).$^{35}$ Children aged $8-15$ years will lead the goal setting at $\mathrm{T} 0$ and scoring of goal attainment, while children aged 6-7 years will complete the process with their parent and assessor. The primary outcome, goal attainment, is defined as attainment of at least one goal to an expected (score of zero) or greater level. While varied interpretations of goal attainment have been used, including averaging the number of goals achieved, recent literature in rehabilitation suggests that the chosen definition reflects a clinically relevant change and allows for appropriate statistical analysis, in that it is not treated as a continuous variable.$^{35-37}$

The secondary outcomes will be assessed as follows:

- Goal attainment at T2 measured using GAS. ${ }^{29}$

- Bike skills acquisition and retention measured using the subscale items related to bike skills in the mobility domain of the functional skills in the Dutch calibration of Paediatric Evaluation of Disability Inventory $\left(\right.$ PEDI-NL) ${ }^{38}$ and the Cycling Skills Checklist ${ }^{39}$ at $\mathrm{T} 1$ and T2. The PEDI is a commonly used scale to measure functional status across the domains of selfcare, mobility and social function in children with disability. As part of its calibration for use in the Netherlands, a subscale was added to the mobility domain involving four levels of bike riding skill. The PEDI-NL has good content and discriminative validity and is reliable in children with disabilities. ${ }^{40}$ The Cycling Skills Checklist is a 20-item checklist of beginner bike skills, where a score out of five is given for each skill. The maximum score for the highest level of bike skills is 100 . It has not been validated in children with CP, however has been used in research with youth with Down syndrome. ${ }^{41}$

- Functional skills measured using the Paediatric Evaluation of Disability Inventory-computer adaptive test $(\mathrm{PEDI}-\mathrm{CAT})^{42}$ at $\mathrm{T} 1$ and T2. The PEDI-CAT comprises a comprehensive item bank of 276 functional activities acquired throughout infancy, childhood and adolescence. The PEDI-CAT measures function in four domains: (1) daily activities; (2) mobility; (3) social/ cognitive and (4) responsibility. It is valid and reliable for use in parents of children with all ages with CP. The content-balanced version of the PEDI-CAT will be used.

- Physical activity behaviour measured using a triaxial accelerometer $^{43}$ and the Physical Activity Questionnaire for Children (PAQ-C) ${ }^{44}$ at T2. Accelerometry is a feasible, reliable and validated method of measuring activity in children and young people with $\mathrm{CP} .{ }^{45}$ The Activ8 will be used as it is able to distinguish cycling as a different type of physical activity from walking, running, standing and sitting. ${ }^{43}$ The Activ8 will be worn by each participant for 7 days at T0 and at T2. PAQ-C is a valid and reliable ${ }^{46}$ self-report 7-day recall assessment of physical activity in children aged 8-20 years.

- Overall self-perception measured with the Pictorial Scale of Perceived Competence and Social Acceptance for Young Children ${ }^{47}$ (aged 6-7 years) or the Self-Perception Profiles for Children ${ }^{48}$ (aged 8-13 years) and Adolescents ${ }^{49}$ (aged 14-15 years) at T1 and T2. These self-perception scales have good validity in children without intellectual impairment. ${ }^{47-49}$

- Self-perceived bike riding competence measured with the bike riding item of the Pictorial Scale of Perceived Movement Skill Competence. ${ }^{50}$ The scale from which this item is drawn has good reliability, and face and construct validity in children. ${ }^{5051}$

- Attendance and involvement for participants in the intervention group during the 3-day programme as recorded by clinicians delivering the intervention group protocol. Any home-based bike skills training during the intervention period in both groups will be recorded by participants and parents each day of the intervention period and each week during the follow-up period in a participant diary. Families will also be asked to assess the involvement of the child of a five-point adjectival scale from minimally involved to very involved in the practice for each day of the 7-day intervention period.

- Quality of life measured by Child Health Utility-Nine Domains (CHU-9D) $)^{52}$ at $\mathrm{T} 1$ and T2. The CHU-9D is a paediatric generic preference-based measure of health-related quality of life. ${ }^{52}$ It consists of a descriptive system and a set of preference weights, giving utility values for each health state described by the descriptive system, allowing for calculation of quality-adjusted life-years for cost-utility analysis. It consists of nine domains and has been validated in 
children aged 7-17 years. Data of resources and time used to deliver the task-specific approach to training bike skills and the parent-led home programme will be collected by clinicians and parents and used for cost-effectiveness analysis.

- Fidelity assessed by examining the adherence of the clinicians and parents to the intervention and comparison group protocols. The amount of time practising bike skills will be measured by participant diaries in both groups. Clinicians will also complete attendance logs for participants in the intervention group and will document adherence to the protocol as reported by the parent on the comparison group phone call. Specific fidelity to the intervention protocol will be by video analysis. One session of the intensive programme per participant will be videoed and analysed for adherence to the protocol using the Motor Learning Strategies Rating Instrument-20 Items. ${ }^{24}$

Participating families will be asked to document any other therapy, health or medical interventions they receive during the study period on the participant diaries.

\section{Exclusion during the study}

All outcome data will be attempted to be collected for all enrolled participants with the exception of those who withdraw consent.

\section{Treatment discontinuation}

Participants in the intervention group or their parents may decide to stop the intervention at any time during the study. If a participant stops the intervention for any reason, all evaluations required for the immediate and final study visit will still be offered to the participant (unless the participant formally withdraws from the study).

\section{Data analysis plan}

\section{Sample size calculation}

Results of a survey conducted by the research team indicate that approximately $25 \%$ of children with CP (GMFCS I-II) had learnt to ride a two-wheel bike in the home environment led by their parents or caregivers, ${ }^{8}$ which is likely to be the key goal of many of the study participants. Within previous studies using GAS to assess the effectiveness of similar interventions in children with $\mathrm{CP}$, the proportion of goals attained or participants who have reached goal attainment has been reported between $66 \%$ and $86 \% .29355354$

Using the results of previous studies, this study is powered to find an absolute difference of $50 \%$ (from $25 \%$ in the home programme/comparison group to $75 \%$ in the intervention group) in the proportion of participants who reach goal attainment following the intervention. Assuming independent observations from individuals, a sample size of 19 in each group (38 in total) would be required to identify a difference in proportions of $50 \%$ with $80 \%$ power (based on a two-sided test with a $5 \%$ level of significance). In this study, participants in the intervention group will receive the intervention in groups. It is likely that the outcomes for participants in the same group will be correlated or clustered hence the sample size has been inflated to account for this correlation. Assuming a small intracluster correlation of 0.1 between individuals within a cluster, and assuming an average cluster size of five, this equates to a design effect of 1.4 , hence we will need to recruit 27 participants per arm (54 participants in total) to obtain the effective sample size of 38 . Finally, we inflate the required sample size to allow for $10 \%$ loss to follow-up, hence we plan to recruit a total of 60 participants (approximately 30 per group).

\section{Statistical analysis}

All statistical analysis will be conducted on an intention-totreat basis where outcome data are available using STATA statistical software V.14. ${ }^{55}$ Descriptive statistics will be used to characterise each group. Logistic regression will be used to assess the effect of providing the novel task-specific intervention compared with the parent-led home programme on the primary outcome, bike-specific goal attainment, adjusted for site as used in the randomisation process. Logistic regression will also be used to compare secondary binary outcomes between each group and linear regression will be used to compare secondary continuous outcomes between groups.

All analyses will be conducted using mixed effects models including a random effect to allow for the clustering of participants within therapy groups in the intervention arm. As a secondary analysis, all analyses will be repeated using a per-protocol analysis. In this analysis, participants in the intervention group who discontinued the intervention prior to completing the 3-day programme will be excluded from the analysis.

Participants will also be excluded from per-protocol analysis in either treatment group if any of the following protocol violations occur following randomisation and during the intervention and follow-up periods:

- Botulinum toxin-A injections to the lower or upper limbs;

- Musculoskeletal surgery or other major surgery that may affect their physical ability;

- Insertion of an intrathecal baclofen pump;

- Occupational therapy or physiotherapy related to training two-wheel bike skills other than the intervention or comparison group protocols.

The economic appraisal will be conducted from a societal perspective. Cost-consequence analysis, including cost-effectiveness analysis and cost-utility analysis, will be carried out by comparing the incremental cost with the incremental benefit. The cost-effectiveness analysis will compare the costs with the primary and secondary outcomes demonstrating significance, and the costutility analysis will compare the costs with the outcomes as measured by the CHU-9D. ${ }^{52}$ The costs associated with resources and time used for each group will be assessed and compared. 


\section{Handling of missing data}

Prior to analysis, the amount of missing data will be explored, along with a comparison of distribution of key variables in individuals with and without missing data. If there is a reasonable amount of missing data and the data summaries suggest that the data are missing at random then all analyses will be presented following multiple imputation for missing data using baseline variables as auxiliary variables. Complete case analysis will also be conducted and reported. In the case there is little missing data, a complete case analysis will form the primary analysis.

\section{Ethics and dissemination}

The trial is registered with the US National Institutes of Health (NCT03003026) and recruitment is ongoing.

Data collected as part of this study will be entered and stored in electronic format on a REDCap secure, web-based database.$^{56}$ All other relevant electronic and paper data files will be stored securely and accessible only to study investigators. Participant confidentiality and privacy will be strictly held in trust by all study personnel.

Given the low risk nature of trial, a data monitoring committee is not required. Adverse events will be recorded from the time the participant signs the informed consent form until the end of the last study visit. Any serious adverse events occurring in a study participant will be reported to all involved ethics committees within 72 hours of occurrence.

This study is being completed as part of RT's Doctor of Philosophy (PhD-physiotherapy) at the University of Melbourne. It will form a major part of her thesis. The results of this study will be submitted to peer-reviewed journals and presented to national and international conferences. Participating families will receive detailed summaries of the results of the study and a brief summary of the results will be distributed through the VCPR biannual newsletter and the CRE-CP e-newsletter/website.

\section{Significance}

This study will contribute to the evidence base regarding the effectiveness of approaches to training bike skills in children with CP for attaining bike-specific goals. Furthermore, the range of secondary outcomes will allow for assessment of the effect of training bike skills on a range of meaningful outcomes for children and their families. The results of the economic evaluation will be used for policy and decision making.

\footnotetext{
Author affiliations

${ }^{1}$ Department of Physiotherapy, University of Melbourne, Melbourne, Australia

${ }^{2}$ Developmental Disability and Rehabilitation Research, Murdoch Children's

Research Institute, Melbourne, Australia

${ }^{3}$ Victorian Paediatric Rehabilitation Service, Monash Children's Hospital, Melbourne, Australia

${ }^{4}$ Neurodevelopment and Disability, The Royal Children's Hospital, Melbourne, Australia

${ }^{5}$ Department of Paediatrics, University of Melbourne, Melbourne, Australia ${ }^{6}$ Clinical Epidemiology and Biostatistics Unit, Murdoch Children's Research Institute, Melbourne, Australia
}

${ }^{7}$ Melbourne Children's Trials Centre, Melbourne Children's Campus, Melbourne, Australia

${ }^{8}$ Deakin Health Economics, Centre for Population Health Research, Deakin University, Geelong, Australia

${ }^{9}$ Victorian Infant Brain Studies, Murdoch Children's Research Institute, Melbourne, Australia

Acknowledgements The authors would like to thank Frances Wright (parent advisor), Professor Andrew Davidson (Director, Melbourne Children's Trials Centre), Associate Professor Adam Scheinberg (State-wide Medical Director, VPRS), Associate Professor Barry Rawicki (Medical Director, VPRS Monash Children's Hospital), Dr Sue Reid (Manager, VCPR) and Professor Dinah Reddihough (Murdoch Children's Research Institute) for their contributions to this protocol and in-kind support of this study.

Contributors All named investigators contributed to the design of this trial protocol, to drafting and revising the manuscript and have approved this version for submission. Lead investigator RT is responsible for all aspects of study conduct with a particular focus on study oversight, recruitment, clinician training, reporting of adverse events, conducting study visits, outcome assessment, data management and statistical methods. Dr ARH, Associate Professor JLMcG and Associate Professor AJS are responsible for selected study procedures (including randomisation allocation) and study oversight. Associate Professor KJL has contributed to statistical methods and will be involved in interpretation of the results. Dr STFS will contribute to economic appraisal. RT will lead the dissemination and translation of results, with contributions from all investigators.

Funding This work was supported by a Physiotherapy Research Foundation Seeding Grant (S16-007), an Australian Government Research Training Program Scholarship (RT), National Health Medical Research Council (NHMRC) Centre of Research Excellence in Cerebral Palsy ID 1057997 (RT, ARH, STFS), Centre of Research Excellence in Newborn Medicine ID 1060733 (AJS), NHMRC Career Development Fellowship ID 1053767 (AJS), Melbourne Children's Campus Clinician Scientist Fellowship (ARH), Murdoch Children's Research Institute (RT, ARH and KJL) and the Victorian Government's Operational Infrastructure Support Program.

Competing interests None declared.

Patient consent Not required.

Ethics approval This study was granted multisite approval by the Human Research and Ethics Committee at the Royal Children's Hospital (\#36209).

Provenance and peer review Not commissioned; externally peer reviewed.

Open Access This is an Open Access article distributed in accordance with the Creative Commons Attribution Non Commercial (CC BY-NC 4.0) license, which permits others to distribute, remix, adapt, build upon this work non-commercially, and license their derivative works on different terms, provided the original work is properly cited and the use is non-commercial. See: http://creativecommons.org/ licenses/by-nc/4.0/

(c) Article author(s) (or their employer(s) unless otherwise stated in the text of the article) 2018. All rights reserved. No commercial use is permitted unless otherwise expressly granted.

\section{REFERENCES}

1. Stanley F, Blair E, Alberman E. How common are the cerebral palsies? Cerebral Palsies: Epidemiology and Causal Pathways. London: MacKeith Press, 2000:22-9.

2. Bax M, Goldstein M, Rosenbaum P, et al. Proposed definition and classification of cerebral palsy, April 2005. Dev Med Child Neurol 2005;47:571-6.

3. Bjornson KF, Belza B, Kartin D, et al. Ambulatory physical activity performance in youth with cerebral palsy and youth who are developing typically. Phys Ther 2007;87:248-57.

4. Fernandes RA, Zanesco A, Sansecso A. Early physical activity promotes lower prevalence of chronic diseases in adulthood. Hypertens Res 2010;33:926-31.

5. Novak I, Mclntyre S, Morgan C, et al. A systematic review of interventions for children with cerebral palsy: state of the evidence. Dev Med Child Neurol 2013;55:885-910.

6. Australian Bureau of Statistics. Children's participation in cultural and leisure activities. 2012 http://www.abs.gov.au/ausstats/abs@.nsf/ Products/4901.0 Apr+2012 Main+Features Recreational+activities? OpenDocument (accessed 18 Feb 2016). 
7. Palisano R, Rosenbaum P, Walter S, et al. Development and reliability of a system to classify gross motor function in children with cerebral palsy. Dev Med Child Neurol 1997;39:214-23.

8. Toovey R, Reid S, Harvey A, et al. Ability of ambulatory children with cerebral palsy to ride a bike and age at skill acquisition. Dev Med Child Neurol 2017;59:395-401.

9. Demuth SK, Knutson LM, Fowler EG. The PEDALS stationary cycling intervention and health-related quality of life in children with cerebral palsy: a randomized controlled trial. Dev Med Child Neurol 2012;54:654-61.

10. Fowler EG, Knutson LM, Demuth SK, et al. Pediatric endurance and limb strengthening (PEDALS) for children with cerebral palsy using stationary cycling: a randomized controlled trial. Phys Ther 2010;90:367-81.

11. Siebert KL, DeMuth SK, Knutson LM, et al. Stationary cycling and children with cerebral palsy: case reports for two participants. Phys Occup Ther Pediatr 2010;30:125-38.

12. Sakzewski L, Ziviani J, Boyd RN. Efficacy of upper limb therapies for unilateral cerebral palsy: a meta-analysis. Pediatrics 2014;133:e17 $5-\mathrm{e} 204$

13. French B, Thomas LH, Leathley MJ, et al. Repetitive task training for improving functional ability after stroke. Cochrane Database Syst Rev 2007:17:CD006073.

14. Hubbard IJ, Parsons MW, Neilson C, et al. Task-specific training: evidence for and translation to clinical practice. Occup Ther Int 2009;16:175-89.

15. Bar-Haim S, Harries N, Nammourah I, et al. Effectiveness of motor learning coaching in children with cerebral palsy: a randomized controlled trial. Clin Rehabil 2010;24:1009-20.

16. Thelen E, Smith L. Theoretical Models of Human Development (chapter 6). John Wiley and Sons. London: Dynamic Systems Theories, 2007.

17. Shumway-Cook A, Woollacott M. Motor control: translating research into clinical practice. 4th ed. Baltimore, MD: Lippincott Williams \& Wilkins, 2012.

18. Bleyenheuft $\mathrm{Y}$, Arnould C, Brandao MB, et al. Hand and Arm Bimanual Intensive Therapy Including Lower Extremity (HABIT-ILE) in children with unilateral spastic cerebral palsy: a randomized trial. Neurorehabil Neural Repair 2015;29:645-57.

19. Kumban W, Amatachaya S, Emasithi A, et al. Effects of task-specific training on functional ability in children with mild to moderate cerebral palsy. Dev Neurorehabil 2013;16:410-7.

20. Toovey R, Bernie C, Harvey AR, et al. Task-specific gross motor skills training for ambulant school-aged children with cerebral palsy: a systematic review. BMJ Paediatrics Open 2017;1:e000078 https:// doi.org/10.1136/bmjpo-2017-000078.

21. Toovey R, Rawicki B, Harvey A. Outcomes of a goal directed intensive bicycle skills group program for children with cerebral palsy: a pilot case series. Australasian Academy of Cerebral Palsy and Developmental Medicine Conference. Adelaide, Australia: Dev Med Child Neurol 2016:60-61.

22. Roberts G, Howard K, Spittle AJ, et al. Rates of early intervention services in very preterm children with developmental disabilities at age 2 years. J Paediatr Child Health 2008;44.

23. Kiresuk TJ, Sherman RE. Goal attainment scaling: A general method for evaluating comprehensive community mental health programs. Community Ment Health J 1968;4:443-53.

24. Ryan J, Levac D, Wright FV. Motor learning strategies rating instrument-20 items (MLSRI-20) instruction manual. Toronto, CA: Holland Bloorview Kids Rehabilitation Hospital, 2016.

25. Thorpe DE, Valvano J. The effects of knowledge of performance and cognitive strategies on motor skill learning in children with cerebral palsy. Pediatr Phys Ther 2002:14:2-15.

26. Thomas RE, Johnston LM, Sakzewski L, et al. Evaluation of group versus individual physiotherapy following lower limb intra-muscular Botulinum Toxin-Type A injections for ambulant children with cerebra palsy: A single-blind randomized comparison trial. Res Dev Disabil 2016;53-54:267-78.

27. Hemayattalab R, Arabameri E, Pourazar M, et al. Effects of selfcontrolled feedback on learning of a throwing task in children with spastic hemiplegic cerebral palsy. Res Dev Disabil 2013;34:2884-9.

28. Löwing K, Bexelius A, Brogren Carlberg E. Activity focused and goal directed therapy for children with cerebral palsy - Do goals make a difference? Disabil Rehabil 2009;31:1808-16.

29. Steenbeek D. Goal attainment scaling in paediatric rehabilitation: Utrecht University, 2010

30. Löwing K, Bexelius A, Carlberg EB. Goal-directed functional therapy: a longitudinal study on gross motor function in children with cerebral palsy. Disabil Rehabil 2010;32:908-16.
31. Kuhlthau KA, Bloom S, Van Cleave J, et al. Evidence for familycentered care for children with special health care needs: a systematic review. Acad Pediatr 2011;11:136-43.

32. Novak I, Cusick A, Lannin N. Occupational therapy home programs for cerebral palsy: double-blind, randomized, controlled trial. Pediatrics 2009;124:e606-e614.

33. Palisano RJ. Validity of goal attainment scaling in infants with motor delays. Phys Ther 1993;73:651-8.

34. Steenbeek D, Ketelaar M, Lindeman E, et al. Interrater reliability of goal attainment scaling in rehabilitation of children with cerebral palsy. Arch Phys Med Rehabil 2010;91:429-35.

35. Steenbeek D, Ketelaar M, Galama K, et al. Goal attainment scaling in paediatric rehabilitation: a critical review of the literature. Dev Med Child Neurol 2007;49:550-6.

36. Toovey R, Harvey AR, McGinley JL, et al. Bike Skills Training for Children With Cerebral Palsy. US National Library of Medicine Clinical Trials Register ID: NCT03003026. ClinicalTrials.gov 2016.

37. Krasny-Pacini A, Evans J, Sohlberg MM, et al. Proposed criteria for appriasing goal attainment scales used as outcome measures in rehabiliation research. Arch Phys Med Rehabil 2016;97:157-70.

38. Wassenberg-Severijnen J, Maas C, Custers J, et al. Standardization of the Dutch 'Pediatric Evaluation of Disability Inventory' (PEDI). Chapter 5, Pediatric Evaluation of Disability Inventory (PEDI): Calibrating the Dutch Version. Utrect University 2005.

39. Halayko J. You Can Ride Too! An Exploration of the Guided Discovery of Two-wheeled Cycling Skills by Youth with Intellectual Disabilities. University of Alberta 2014.

40. Custers J, et al. Discriminative validity of the Dutch PEDI. Arch Phys Med Rehabil 2002;83:1437-41.

41. Halayko J, Magill-Evans J, Smith V, et al. Enabling 2-wheeled cycling for youth with Down Syndrome. Pediatr Phys Ther 2016;28:224-30.

42. Haley SM, Coster WJ, Dumas HM, et al. Pediatric Evaluation of Disability Inventory Computer Adapative Test - Development, Standardization and Administration Manual. http://www.pedicat.com. 2012 (accessed Jul 2016).

43. Activ8 (TM) physical activity monitor. https://www.activ8all.com/2015 (accessed 17 Jul 2016).

44. Crocker PR, Bailey DA, Faulkner RA, et al. Measuring general levels of physical activity: preliminary evidence for the Physical Activity Questionnaire for Older Children. Med Sci Sports Exerc 1997;29:1344-9.

45. Gorter JW, Noorduyn SG, Obeid J, et al. Accelerometry: a feasible method to quantify physical activity in ambulatory and nonambulatory adolescents with cerebral palsy. Int J Pediatr 2012;2012:1-6.

46. Janz KF, Lutuchy EM, Wenthe P, et al. Measuring activity in children and adolescents using self-report: PAQ-C and PAQ-A. Med Sci Sports Exerc 2008;40:767-72.

47. Harter S, Pike R. The pictoral scale of percevied competence and social acceptance for young children: manual: University of Denver, 1983. https://portfolio.du.edu/SusanHarter/page/44342 (accessed July 2016).

48. Harter S. Self-Perception profile for children: Unviersity of Denver, 2012. https://portfolio.du.edu/SusanHarter/page/44210 (accessed July 2016).

49. Harter S. Self-Perception profile for adolescents: University of Denve, 2012. https://portfolio.du.edu/SusanHarter/page/44210 (accessed July 2016).

50. Barnett LM, Ridgers ND, Zask A, et al. Face validity and reliability of a pictorial instrument for assessing fundamental movement skill perceived competence in young children. J Sci Med Sport 2015;18:98-102.

51. Barnett LM, Vazou S, Abbott G, et al. Construct validity of the pictorial scale of Perceived Movement Skill Competence. Psychol Sport Exerc 2016;22:294-302.

52. Stevens K. Assessing the performance of a new generic measure of health-related quality of life for children and refining it for use in health state valuation. Appl Health Econ Health Policy 2011;9:157-69.

53. Ahl LE, Johansson E, Granat T, et al. Functional therapy for children with cerebral palsy: an ecological approach. Dev Med Child Neurol 2005; $47: 613-9$.

54. Sorsdahl AB, Moe-Nilssen R, Kaale HK, et al. Change in basic motor abilities, quality of movement and everyday activities following intensive, goal-directed, activity-focused physiotherapy in a group setting for children with cerebral palsy. BMC Pediatr 2010;10:26.

55. StataCorp. Stata Statistical Software: Release 14. College Station, TX, 2015.

56. Harris PA, Taylor R, Thielke R, et al. Research electronic data capture (REDCap) - a metadata-driven methodology and workflow process for providing translational research informatics support. J Biomed Inform 2009;42:377-81. 